\title{
Assessing dynamic changes in flavor-related compounds during durian fruit ripening using metabolome and transcriptome analyses
}

\section{Supaart Sirikantaramas ( $\sim$ supaart.s@chula.ac.th )}

Chulalongkorn University https://orcid.org/0000-0003-0330-0845

\section{Lalida Sangpong}

Chulalongkorn University

\section{Gholamreza Khaksar}

Chulalongkorn University

\section{Pinnapat Pinsorn}

Chulalongkorn University

\section{Akira Oikawa}

Yamagata University

\section{Ryosuke Sasaki}

3RIKEN Center for Sustainable Resource Science

Erban Alexander

Max Planck Society

Mutsumi Watanabe

Max Planck Institute

Karan Wangpaiboon

Chulalongkorn University

\section{Takayuki Tohge}

Max Planck Institute

Kopka Joachim

Max Planck Institute for Molecular Plant Physiology

\section{Rainer Hoefgen}

Max Planck Institute of Molecular Plant Physiology https://orcid.org/0000-0001-8590-9800

\section{Kazuki Saito}

RIKEN https://orcid.org/0000-0001-6310-5342

\section{Article}

Keywords: durian, ripening-associated metabolites, transcriptome analysis 
Posted Date: March 24th, 2021

DOI: https://doi.org/10.21203/rs.3.rs-125662/v1

License: (c) (i) This work is licensed under a Creative Commons Attribution 4.0 International License. Read Full License 


\section{Abstract}

Durian is an economically important fruit of Southeast Asia. There is, however, a lack of in-depth information on the alteration of metabolic networks during its ripening. Here, we annotated 94 ripeningassociated metabolites from the pulp of durian cv. Monthong fruit at unripe and ripe stages, using capillary electrophoresis- and gas chromatography- time-of-flight mass spectrometry, specifically focused on flavor-related metabolites. During ripening, sucrose content was found to be increased. Change in raffinose-family sugars is reported herein for the first time. The contents of malate and succinate increased, while those of citrate, an abundant organic acid, were unchanged. Notably, most amino acids increased, including isoleucine, leucine, and valine, whereas aspartate decreased, and glutamate was unchanged. Furthermore, transcriptome analysis was performed to support the dynamic changes in several flavor-related pathways, and for the identification of key candidate genes. Taken together, our results could be exploited for developing durian metabolic/genetic markers in the future.

\section{Introduction}

Durian (Durio zibethinus L.) is a highly economically valuable fruit endemic to Southeast Asia, which has recently begun to be distributed globally. Owing to the increasing demand for durian, the price and production of the fruit tend to increase every year. Durian is well known for its outstanding flavor, described as an overpowering sweet taste, with a sweet, fruity odor resulting from high contents of starch, sugar, and saturated fatty acids in the ripe pulp ${ }^{1}$.More interestingly, ripe durian pulp comprises various bioactive compounds, including carotenoids, flavonoids, and polyphenols, associated with its high antioxidant properties ${ }^{2}$, suggesting that consumption of durian may provide potential health benefits. All the characteristics of the edible durian are contributed by the ripening process.

Fruit ripening is a complex and coordinated developmental process associated with pronounced molecular and biochemical changes. The climacteric fruits, including durians, are usually harvested at the commercial maturity stage. Afterwards, the fruits undergo a postharvest ripening process, during which the rates of respiration and ethylene production are dramatically increased. During this process, stored carbohydrates are broken down to sugars, and acidity is reduced alongside the increase in flavor and aroma volatiles ${ }^{3}$. Moreover, modifications of amino acids and organic acids are linked with fruit aroma by acting as precursors for the biosynthesis of volatile compounds involved in aroma formation ${ }^{4}$. Taken together, these modifications are the key contributors to fruit flavor.

Over the past decade, the advent of 'omics' approaches has significantly contributed to the identification of functional metabolites in plant metabolism (primary and secondary) during fruit development. Metabolomics has been successfully used to identify primary metabolites, including sugars, organic acids, amino acids, and other related compounds, and to provide an understanding of whole metabolic alteration during development and ripening of fruits, such as peach, tomato, strawberry, and grape 5 . Moreover, metabolomics, coupled with transcriptomics, provides information on major metabolic networks and candidate genes controlling the underlying processes; integration of these data with 
genomics provides new insights into the major metabolic variations and their genetic and biochemical control during development. Such studies have widely been performed on tomato, a popular model fruit, and documented how breeding and genetic selection globally altered tomato fruit metabolite content ${ }^{6}$.

Metabolic profiles of durian pulp at the ripe stage have been investigated using capillary electrophoresistime of flight/mass spectrometry (CE-TOF/MS) ${ }^{7}$. Cultivar-dependent metabolites associated with the sensory traits of durian fruit pulps, such as odor-related (cysteine, leucine) and ripening-associated (aminocyclopropane carboxylate) metabolites, are of utmost importance. In 2017, the draft genome of durian was first published, and genome data integrated with transcriptome data identified methionine- $\gamma^{-}$ lyase as a key gene involved in controlling sulfur volatile compound production in durian pulp ${ }^{8}$. The genome data led to further studies to better understand the durian ripening process. Subsequently, a genome-wide analysis of the Dof (DNA binding with one finger) transcription factor family identified 24 Dofs (DzDofs), among which 15 were expressed in the fruit pulp. Functional characterization of DzDof2.2 suggested that it has a role in fruit ripening by regulating auxin biosynthesis and auxin-ethylene crosstalk ${ }^{9}$. In addition, a member of the auxin response factor (ARF) transcription factor family was identified, showing that DzARF2A mediates durian fruit ripening through transcriptional regulation of ethylene biosynthesis genes ${ }^{10}$. In this regard, omics analyses can be powerful tools to improve our limited understanding of mechanisms underlying the durian postharvest ripening process, especially the changes in primary metabolites. In this study, we coupled metabolomics and transcriptomics to investigate the metabolic profile of the Monthong cultivar, the most widely cultivated durian in Thailand, during post-harvest ripening using capillary electrophoresis (CE), gas chromatography (GC), time-of-flight mass spectrometry (TOF/MS) to elucidate the ripening-associated metabolites. Our findings provide comprehensive information on metabolic shifts during post-harvest ripening of durian fruit pulp. The identification of ripening-associated metabolites, which contribute to its unique flavors, may be further exploited in durian breeding programs to develop cultivars with altered sensory characteristics and/or enhanced nutritional value.

\section{Results And Discussion}

\section{An overview on metabolites involved in durian fruit ripening}

The changes in the underlying mechanisms during the ripening process contribute to durian fruit characteristics. To study the alterations of primary metabolites in these related mechanisms, we performed metabolome analysis in durian cv. Monthong pulp at the unripe and ripe stages. CE-TOF/MS and GC-TOF/MS techniques were used for comprehensive analysis of primary metabolites, which identified 167 and 56 metabolites, respectively. Among these metabolites, 28 were annotated using both techniques (Supplementary Table S1, Supplementary Fig. S1). In total, 195 annotated metabolites were obtained and used for further analysis. Our annotation showed that results from each technique validated those from other techniques because the intensities of the same metabolites exhibited similar changes during the ripening period (Supplementary Table S1). It is known that both CE-TOF/MS and GC- 
TOF/MS annotate primary metabolites of slightly different kinds. CE-TOF/MS can separate a wider range of charged metabolites, including amino acids and organic acids, while GC-TOF/MS is more dependent on volatility and can identify uncharged metabolites, such as sugar and sugar derivatives.

Metabolites were further analyzed using the web-based tool MetaboAnalyst 4.0. From the PCA plot, the first two components on the $x$ - and $y$-axes represented the total variance of the data at $42.5 \%$ and $33.2 \%$, respectively. Unripe durian samples were clearly separated from the ripe groups by principal component 1 (Fig. 1A). As expected, the results showed that ripening greatly influences the metabolic change at the biochemical level and leads to physiological changes, such as the expression of flavor and softening of the edible ripe fruit. This phenomenon is likewise observed in other fruit species, such as peach, including strawberry, tomato, and grape 5,11 , constituted the different fruit models. To obtain the relevant biological pathways, Metabolomic Pathway Analysis (MetPA) was used. Of 66 enriched KEGG biological pathways, 30 metabolic pathways were perturbed (Supplementary Table S2). Some of the significant altered metabolic pathways, such as the TCA cycle, amino acid metabolism, and starch and sucrose metabolism, are primary metabolic pathways and are possibly involved in flavor precursor biosynthesis (Fig. 1B).

\section{Ripening-associated metabolites contributing to durian flavor}

Out of 195 annotated metabolites, 94 metabolites in durian pulp were significantly altered between the unripe and ripe stages. The ripening-associated metabolites were classified into six groups, including proteinogenic amino acids, non-proteinogenic amino acids, sugar and sugar derivatives, polyamine, organic acid, and nucleotide and nucleotide derivatives. All others were placed in a miscellaneous group (Fig. 2a, 2b, Supplementary Table S3).

Sugar is an important metabolite group responsible for sweetness in fruits. Previously, sucrose, fructose, glucose, and maltose were found to be increased in ripe durian pulp samples ${ }^{1}$. Using GC-TOF/MS, we confirmed that these four major sugars and a newly annotated sugar, raffinose, were significantly increased during ripening with the $\log _{2} \mathrm{FC}$ ranging between 1.16. and 4.84 (Fig. 2), while a newly annotated 1-kestose was unchanged. Since sugars were only annotated from GC-TOF/MS in this work, we applied HPAEC-PAD analysis to validate and confirm the result (Fig. 3). According to the results, the contents of all four major sugars agreed with GC-TOF/MS. However, the content of 1-kestose was significantly increased, while the content of raffinose remained unchanged during the ripening period (Fig. 3). Disagreements between the two methods could be obtained when the concentrations in the samples were very low, as raffinose and 1-kestose were in this case. When using GC-MS, it is suggested that optimum derivatization and separation are needed before measuring sugars in each individual plant extract $^{12}$. Therefore, in our analysis, the quantification from targeted HPAEC-PAD analysis is more reliable than the untargeted analysis, which can lead to the discovery of novel metabolites. Fruits can accumulate different types of sugars, but sucrose is the primary storage sugar in many fruits, such as melon, banana, peach, and strawberry ${ }^{13}$. Notably, durian pulp contains over $50 \%$ sucrose by dry weight (Fig. 3), which makes it the highest sucrose-accumulating fruit among those reported. Our study showed that sucrose is an important flavor-providing metabolite in ripe durian pulp. 
In addition, we identified two ripening-associated sugar alcohols found at low concentration related to raffinose, galactinol and myo-inositol (Fig. 2). Galactinol content decreased during ripening, which was in agreement with the two analytical methods (Fig. 3), whereas myo-inositol content remained unchanged, as confirmed by HPAEC-PAD. Raffinose and myo-inositol were synthesized from galactinol. The decrease in galactinol during the ripening period suggested that it might be stored before ripening and then converted to the downstream sugars later. The accumulation of raffinose-family sugars is quite different between various fruit ${ }^{14}$, and their role in fruits has not been studied much to date; however, they might be involved in stress tolerance during ripening. Furthermore, we found an increase in sugar phosphates, including glucosamine-6-phosphate, $N$-acetyl-D-galactosamine-6-phosphate, lactose-1-phosphate, sucrose-6-phosphate, and trehalose-6-phosphate in ripe durian pulp with $\log _{2}$ FC ranging between 1.34 and 2.09 (Fig. 2). Sugar phosphates are intermediates in different biological pathways. In addition, they also act as signaling molecules. One interesting metabolite is trehalose-6-phosphate, which is an intermediate for trehalose biosynthesis. It is an important signaling molecule for sucrose consumption and a negative feedback molecule for sucrose biosynthesis in plants ${ }^{15}$. Therefore, the increase in trehalose-6-phosphate in durian pulp possibly helps to control the sucrose content and supports trehalose content at the same time.

We detected 21 ripening-related organic acids in the durian pulp. Of these, the levels of 19 organic acids significantly increased during durian ripening, with $\log _{2}$ FC ranging between $1.38-10.33$, while those of 2isopropylmalate and threonate decreased ( $\log _{2} \mathrm{FC}=-1.25$ and -2.56 , respectively) (Fig. 2$)$. There were five ripening-related organic acids, namely cis-aconitate, fumarate, malate, pyruvate, and succinate, that participated in central carbon pathways. Malate and succinate were the highest among others (Supplementary Table S1), which was consistent with the results in the ripe durian pulps from other cultivars $^{16}$. Another abundant organic acid was citrate, and its content was not significantly changed during the ripening period (Supplementary Table S1). Therefore, malate and succinate probably contribute to sour taste in the ripe fruit, even if the sweet taste is dominant in the pulp, due to high sugar composition. In addition, we also detected a significant change in 4-methyl-2-oxovalerate and 2isopropylmalate (Fig. 2), which are two important intermediates in branched-chain amino acid metabolism that will be elaborated in the section below.

Amino acids serve as important flavor precursors in many fruits, including durian. We detected 31 ripening-associated amino acids, three amino acids that were unchanged: asparagine, glutamate, and threonine. Levels of 15 proteinogenic and 14 non-proteinogenic amino acids increased during durian ripening with $\log _{2} \mathrm{FC}$ ranging between 1.34 - 5.65 and between 1.4 - 9.15, respectively, while only aspartate and ornithine decreased ( $\log _{2} \mathrm{FC}=-1.36$ and -5.64 , respectively) (Fig. 2). Durian pulp releases sulfuryl odor during ripening. The odor is associated with amino acids in sulfur-related pathways. Notably, cysteine is the most modulated amino acid in the pathway (Fig. 2, Supplementary Table S3) and is an important precursor for the biosynthesis of methionine, another sulfur-containing amino acid. We found that methionine levels slightly increased in ripening durian pulp (Fig. 2, Supplementary Table S3). Methionine is a central metabolite of the sulfur pathway and ethylene biosynthesis, in which ethylene is 
an important compound for climacteric ripening. Therefore, this may be the reason for the slight changes in methionine content during ripening. In addition, we also found the accumulation of serine and homoserine (Fig. 2), which are intermediates in the sulfur pathway, supporting the involvement of the pathway during the ripening process.

In addition to sulfuryl odor, durian pulp also exhibits fruity aroma during ripening. Branched-chain amino acids, including isoleucine, leucine, and valine, are precursors for alcohols, esters, aldehydes, and lipidderived volatiles ${ }^{17}$, which are associated with the fruity smells. We found a significant increase in the levels of these three amino acids in ripe durian pulp (Fig. 2). Interestingly, the increase in isoleucine enhances the biosynthesis of 3-methylbutyl alcohols, 2-methylbutyl alcohols, and their ester derivatives, while the increase in valine enhances 2-methylpropyl alcohol derivatives ${ }^{18}$. These volatiles can also be detected in the durian and provide the fruit with a fruity odor ${ }^{19}$. Therefore, it implies that the accumulation of branched-chain amino acids during ripening is important for the biosynthesis of volatile compounds in durian pulp. Moreover, the altered composition of branched-chain amino acids may lead to differences in proportions of volatile compounds and may cause different aroma among durian cultivars.

For the aromatic amino acid pathway, phenylalanine, tryptophan, and tyrosine are ripening-related amino acids in durian fruit (Fig. 2). These amino acids are precursors for the biosynthesis of volatile compounds. For example, phenylalanine increases the production of aldehyde- and ester- type aromatic volatiles ${ }^{4}$, and some phenyl volatiles are also found in durians ${ }^{19}$. Furthermore, aromatic amino acids are precursors for secondary metabolites such as flavonoids, alkaloids, indoles, etc. Interestingly, durian pulp contains a high amount of flavonoids at the ripe stage, which positively correlates with the high antioxidant activity of the pulp ${ }^{20}$. Accumulation of these amino acids may be important for durian pulp to synthesize secondary metabolites during ripening.

Aspartate was the only proteinogenic amino acid whose levels decreased during ripening (Fig. 2). It can be directly used for the biosynthesis of glutamate and asparagine. Moreover, it is involved in the biosynthesis of lysine, methionine, isoleucine, leucine, and valine, whose contents increased during ripening. Therefore, the decrease in aspartate levels during ripening could result from its usage for biosynthesis of other amino acids. Glutamate is a highly abundant amino acid that provides umami taste in durian pulp $p^{7}$. We found that the glutamate content remained constant during the ripening period (Supplementary Table S1). Glutamate can be converted to other metabolites, such as glutamine, arginine, proline, and $\mathrm{GABA}^{21}$. In fact, we observed increased levels of proline and hydroxyproline during ripening. Until now, the roles of these two metabolites in fruits have not been well understood. Previous studies have shown that accumulation of proline is involved in the nitrogen sink mechanism, and it helps to increase the fruit quality traits and yield ${ }^{22}$. Regarding the precursors for polyamine biosynthesis, we found that the arginine content was unchanged (Supplementary Table S1), while ornithine content decreased during the ripening period (Fig. 2). Both amino acids can be converted to putrescine, a central metabolite of the polyamine pathway. 
For polyamine, we found significant increases in $N^{8}$-acetylspermidine and putrescine, while spermidine and spermine were unchanged and decreased, respectively (Fig. 2, Supplementary Table S1). Polyamines are associated with abiotic stress responses in fruits ${ }^{23}$. Furthermore, previous studies have shown that they are also involved in the modulation of fruit ripening by intricate crosstalk with the ethylene pathway ${ }^{24}$. The role of polyamines in durian pulp still requires further investigation, and their accumulation might affect ripening behavior among various durian cultivars. Polyamines are also precursors for GABA biosynthesis. Interestingly, GABA was highly enriched in ripe durian pulp (Supplementary Table S3, Fig. 2). GABA can provide a bitter taste to the fruit. Although the role of GABA accumulation in fruit has not been clearly ascertained, previous studies have shown that it is associated with abiotic stress responses in strawberry ${ }^{25}$, tomato ${ }^{26}$, and apple ${ }^{27}$. It has been shown that these fruits accumulate high GABA content under elevated $\mathrm{CO}_{2}$ and low $\mathrm{O}_{2}$. Therefore, the role of GABA in durian might also be involved in stress response. Further studies in durian may help to promote its market value by developing appropriate storage conditions for higher GABA content in the future.

The levels of nine nucleotide derivatives increased during ripening, with $\log _{2} \mathrm{FC}$ ranging between 1.32 8.28 (Fig. 2), while those of cytidine diphosphate (CDP) and deoxyguanosine decreased (Log2 FC = -2.06 and -2.18, respectively). To our knowledge, 4 of 11 nucleotide derivatives are known to be involved in fruit flavor. AMP, IMP, and GMP accumulation is associated with enhancing of the umami taste in tomato ${ }^{28}$. In addition, we also detected additional 17 miscellaneous metabolites with significantly altered levels during durian ripening with $\log _{2} \mathrm{FC}$ ranging between -2.18 and 8.45 (Fig. 2). Some of these metabolites are food supplements in the durian pulp, such as gluconate and pyridoxamine, so called vitamin B group ${ }^{7}$.

\section{Transcriptome analysis of durian cv. Monthong during ripening}

The metabolome data provided us only the ripening-associated metabolites and the related pathways associated with durian flavor. Therefore, to deepen and more comprehensively understand the modification of the pathways at the biochemical level, we integrated transcriptome analysis to study the expression of candidate genes in associated pathways.

Approximately 10 - 16 million clean reads from unripe and ripe Monthong were generated. The clean reads were then used to perform de novo assembly, which generated 164,618 unigenes with an average length of $1,082.68 \mathrm{bp}$, and total reads of each library were mapped to the de novo assembled transcriptome (Supplementary Table S4). The annotation results of unigenes are shown in Supplementary Table S5. Differentially expressed gene analysis identified a total of 18,616 DEGs (Supplementary Table S6) with 8,145 up-regulated genes including the key ethylene biosynthetic genes (1-aminocyclopropane-1-carboxylate synthase and 1-aminocyclopropane-1-carboxylate oxidase) (Supplementary Table S7), and 10,471 down-regulated genes. DEGs were then used to perform GO analysis, and most of the GO terms were assigned to biological processes. Within biological processes, the largest proportion was in protein modification by small protein conjugation and ubiquitin-dependent protein catabolic process categories, respectively. Within molecular function, the largest proportion was in 
the ATPase activity category. Lastly, within the cellular compartment, the largest proportion was in the host cell nucleus category (Supplementary Fig. S3). To reveal the biological pathways associated with DEGs data, DEGs were assigned to KEGG. The results showed that the DEGs participated in 144 biological pathways. The top five pathways are purine metabolism, thiamine metabolism, starch and sucrose metabolism, glycolysis/gluconeogenesis, and pyruvate metabolism (Supplementary Fig. S4). This result is consistent to the metabolic pathway analysis where the change of nucleotide metabolism, sugar metabolism, and central carbon metabolism was highly associated to durian ripening process.

To validate the DEGs identified from RNA-seq results, we randomly selected 11 key genes ( $A L D H$ (LOC111284558), ADC (LOC111308806), CM (LOC111286253), GABA-TK(LOC111305652), GAD (LOC111281776), BCAT (LOC111305652), MDH(LOC111293205), NADP-MEs (LOC111286064), OAT (LOC111278083), PFK (LOC111285266), and TrpAB (LOC111287933)) from various metabolic pathways possibly related to durian flavor, and their expression levels were analyzed by RT-qPCR using Monthong cDNA at the unripe and ripe stages. According to the RT-qPCR analysis, the expression results of all selected genes agreed with results obtained from RNA-seq analysis showing the reliability of the RNA-seq method for expression quantitation (Fig. 4).

\section{Identification of candidate genes in metabolic pathways contributing to durian flavor}

Our results demonstrated that sugar and central carbon pathways are important pathways during the ripening process (Supplementary Fig. S4). High sugar accumulation promotes sweet taste in ripe durian pulp. Alternatively, sugars are metabolized into the central carbon pathway, which not only produces cellular energy, but also provides organic acids for fruit acidic taste. In this section, we focused on the modulation of the central carbon pathway and its connection to flavor-related pathways. Therefore, a metabolic network was constructed to elucidate the relationship between changes in metabolite levels and candidate genes (Fig. 5). Information related to candidate genes, such as locus number and expression level, are provided in Supplementary Table S7.

\section{Central carbon pathway}

We identified genes in the glycolytic pathway and found the upregulation of hexokinase $(H K)$, phosphofructokinase (PFK), and pyruvate kinase (PK) (Fig. 5). These three genes control the rate-limiting steps of the glycolytic pathway in fruit ${ }^{29}$. The gene expression is consistent with the accumulation of pyruvate, which is the final product of the pathway (Fig. 5), showing the activation of glycolysis during durian ripening. Afterward, pyruvate is converted to acetyl-CoA and enters the TCA cycle by pyruvate dehydrogenase $(P D H)$, which was also found to be upregulated in the ripe durian pulp (Fig. 5). The first step of the TCA cycle is to produce citrate, which is controlled by citrate synthase (CS). Although CS was upregulated, the content of citrate remained constant during ripening (Fig. 5), suggesting that the rate of citrate biosynthesis is probably equal to citrate consumption. We also observed the upregulation of other genes in the TCA cycle, including isocitrate dehydrogenase (IDH), a-ketoglutarate dehydrogenase (2$O G D H)$, fumarase (FUM), and malate dehydrogenase $(M D H)$, together with the upregulated metabolites, 
isocitrate, succinate, fumarate, and malate (Fig. 5), showing the activation of the TCA cycle, supporting citrate biosynthesis during ripening. Citrate usage probably goes through glutamate metabolism through a-ketoglutarate. We found that the content of a-ketoglutarate was unchanged during the ripening period. Notably, the gene for its biosynthesis, IDH, and the gene for its degradation, 2-OGDH, and glutamate dehydrogenase $(G D H)$, were upregulated (Fig. 5). This result suggests that a-ketoglutarate content remains constant because it is converted to the TCA cycle by 2-OGDH and bypassed to glutamate metabolism by GDH. Similar observations were found in other fruits, such as tomato ${ }^{30}$, showing that the change in citrate is correlated to a-ketoglutarate content during ripening and salt-stress treatment. Although some metabolites in this pathway do not provide flavor to ripe durian, they serve as important precursors for the biosynthesis of other flavor-related compounds, which will be discussed later.

\section{Glutamate and glutamate-derived metabolic pathways}

Glutamate, an amino acid providing umami taste, is linked to the TCA cycle via a-ketoglutarate, which is controlled by $G D H$, and we found that the gene was upregulated during the period of ripening (Fig. 5, Supplementary Table S7). Additionally, there are three routes for glutamate biosynthesis, including aspartate aminotransferase (AST), alanine aminotransferase (ALT), and glutamate synthase (GOGAT). We found that only AST and GOGAT were upregulated during the period of ripening (Fig. 5). Notably, the route from AST (LOC111284590) contained the highest $\log _{2}$ FC (4.83) and RPKM expression value among the other routes (Supplementary Table S7). Therefore, such a route is highly activated for glutamate biosynthesis during ripening. Glutamate interconverts with aspartate, and this step is controlled by $A S T$. We found that the decrease in aspartate content during durian ripening correlated with the upregulation of $A S T$ (Fig. 5), suggesting a transfer of amino group from aspartate toward glutamate.

Although glutamate biosynthesis was highly activated, its content remained unchanged during the period of ripening (Fig. 5), implying an equal rate of biosynthesis and consumption. Interestingly, we found upregulation of several genes related to glutamate conversion. GS was correlated with an increase in glutamine content (Fig. 5). In addition, we also found the upregulation of nitrate reductase (NR) (Supplementary Table S7). Based on the upregulation of these genes, the activation of nitrogen assimilation, leading to enhanced fruit flavor by modulating levels of amino acid and soluble sugars can be suggested. Glutamate can also be sequentially converted to $\Delta^{1}$-pyrroline-5-carboxylate and proline by enzymes encoded from $\Delta^{1}$-pyrroline-5-carboxylate synthetase (P5CS) and $\Delta^{7}$-pyrroline-5-carboxylate reductase (P5CR), respectively. Only one isoform of P5CS and P5CR were found in our transcriptome data, and they were both downregulated during durian ripening, which was not consistent with increased proline content (Fig. 5). Previous report showed that proline content may not be correlated to the expression of P5CR in developing tissues of Arabidopsis ${ }^{31}$. Therefore, these durian P5CS and P5CR could still be the only candidate genes of proline metabolism in the pulp. We assumed that although both genes were downregulated during ripening, they might still encode enough enzymes that catalyze the reactions and produce proline. In addition, the up-accumulation of proline might be partially caused by a 
lower rate in proline degradation since we observed the downregulation of a gene encoding proline dehydrogenase, an enzyme involved in proline catabolism (Supplementary Table S7).

Furthermore, glutamate can be used for the biosynthesis of ornithine and arginine, which are precursors for polyamine biosynthesis. These two amino acids can later be converted to putrescine, a precursor for further polyamine production. We propose that the primary route for polyamine biosynthesis of durian pulp is arginine because the key gene arginine decarboxylase $(A D C)$ was upregulated and highly expressed during durian ripening (Fig. 5), while the expression level of ornithine decarboxylase (ODC), controlling the reaction from ornithine to putrescine, was not significantly changed. The arginine route has been found to be a major pathway for polyamine biosynthesis in other fruits such as apple and tomato $^{32}$. For the polyamine pathway, spermidine synthase (SPDS) and spermine synthase (SPMS) control steps that convert putrescine to spermidine and spermine, respectively. We found upregulation of SPDS and SPMS during durian ripening (Fig. 5), which were not correlated with spermidine and spermine content. Spermidine is either converted to 4-aminobutyraldehyde or is used for spermine biosynthesis. Moreover, spermine can be degraded into $\beta$-alanine metabolism. Therefore, these probably are the reasons for the unchanged spermidine content and the decreased spermine content during the ripening period.

GABA is a bioactive compound that its biosynthetic pathway bypasses from a-ketoglutarate. Interestingly, we found a significant increase in GABA levels in the ripe durian pulp (Fig. 2). There are two main GABA biosynthetic pathways in fruits, which are GABA shunts and alternative pathways from polyamines. For the GABA shunt, we found the upregulation of glutamate decarboxylase $(G A D)$ in ripening durian pulp, which positively correlated with GABA content (Fig. 5). GABA shunts are the major pathway in many fruits. $G A D$ is the key gene that controls the conversion from glutamate to GABA in this pathway. In addition, we found the upregulation of several genes in polyamine-derived-GABA biosynthesis, including polyamine oxidase $(P A O)$ and 4-aminobutanal dehydrogenase ( $A L D H 10)$. Therefore, our gene expression results supported that GABA production during durian ripening was contributed by the two pathways.

\section{Branched-chain amino acid pathway}

An overpowering smell is a well-known characteristic of ripe durian. Besides the sulfuryl aroma, ripe durian also contains a sweet, fruity aroma, and the volatiles providing such aroma are associated with branched-chain amino acid metabolism. Interestingly, we observed high upregulation of threonine ammonia-lyase $(T A L)\left(\log _{2} \mathrm{FC}=5.52\right)$ (Supplementary Table S7), which converts threonine to 2oxobutanoate, an important intermediate for BCAA biosynthesis, during durian ripening (Fig. 5). Moreover, we also observed high upregulation of genes in the BCAA pathway, such as branched-chain amino acid aminotransferase $(B C A T)\left(\log _{2} \mathrm{FC}=6.32\right)$ (Supplementary Table S7), which is a key regulatory gene controlling branched-chain amino acid biosynthesis and degradation ${ }^{18,33}$, supporting the activation of this pathway. In addition, branched-chain amino acids are precursors for volatile ester production. Notably, the upregulation of 3-methyl-2-oxobutanoate dehydrogenase (BCKDHA) and long-chain-enoyl- 
COA hydratase (EHHADH), key genes in pathways connected to branched-chain amino acid degradation ${ }^{34}$, was observed during durian ripening (Fig. 5, Supplementary Table S7). Our results support the hypothesis that branched-chain amino acid biosynthesis is upregulated during durian ripening, and these amino acids are possibly used for volatile ester production, providing aroma to the ripe durian.

In conclusion, our findings provide fundamental knowledge for future durian molecular studies that will allow comparison of the candidate gene expression and their related metabolites among different durian cultivars, thereby assisting in developing molecular markers for durian breeding in the future.

\section{Materials And Methods}

\section{Plant materials and sample preparation}

Durian cv. Monthong was harvested from an orchard in the Trat province of Thailand in 2016. The three stages of the durian fruit used in this study are as follows: unripe, midripe, and ripe. For the unripe stage, the fruit were harvested at the commercially mature stage of 105 days after anthesis. The fruit at midripe and ripe stages were harvested at the commercially mature stage and kept at room temperature $\left(28^{\circ} \mathrm{C}\right)$ until reaching a firmness of $3.4 \pm 0.81 \mathrm{~N}$ and $1.55 \pm 0.45 \mathrm{~N}$, respectively ${ }^{9}$. At each stage, durian pulp was collected from the central seed of each locule, immediately frozen in liquid nitrogen, and ground into powder. The powder was either freeze-dried for CE-TOF/MS, GS-TOF/MS, and high-performance anionexchange chromatography with pulsed amperometric detection (HPAEC-PAD) analyses or stored at $-80^{\circ} \mathrm{C}$ for RNA extraction. Another seed from a different locule of the same fruit was used to analyze firmness, using a texture analyzer, to ensure that samples of the same stage from different cultivars were under identical conditions.

\section{Metabolite analysis using CE-TOF/MS}

Metabolite extraction was performed according to our previous method ${ }^{7}$. In brief, the freeze-dried sample (5 mg) was mixed with methionine sulfone and camphor 10-sulfonic acid as internal standards (SigmaAldrich, St. Louis, MO, USA). CE-TOF/MS analysis was then performed as previously described ${ }^{35}$. Methionine sulfone and camphor 10-sulfonic acid were used for cation and anion analyses, respectively, and their peak areas were used to normalize the peak areas of metabolites, providing the relative intensity of each metabolite. In addition, the CE-TOF/MS data of the durian at the ripe stage harvested in 2016 were retrieved from our previous paper ${ }^{7}$.

\section{Metabolite analysis using GC-TOF/MS}

Metabolite extraction, derivatization, and chromatography data processing were performed according to the methods described previously ${ }^{36}$. In brief, the freeze-dried sample $(10 \mathrm{mg})$ was mixed with $360 \mu \mathrm{L}$ methanol-mix that includes ${ }^{13} \mathrm{C}_{6}$-sorbitol as an internal standard, followed by $200 \mu \mathrm{L}$ chloroform and 400 $\mu \mathrm{L}$ water that are added for phase separation. Solvents were of highest available purity (Merck, Darmstadt, Germany). Samples were vortexed and agitated $15 \mathrm{~min}$ at $70^{\circ} \mathrm{C}$ after adding the methanol- 
mix, incubated $5 \mathrm{~min}$ at $37^{\circ} \mathrm{C}$ after chloroform addition, and thoroughly vortexed after water addition. Phase separation was induced by centrifugation. The upper polar phase $(80 \mu \mathrm{L})$ was dried. Each sample was chemically derivatized using methoxyamine hydrochloride in pyridine and BSTFA (Macherey-Nagel, Düren, Germany) as described ${ }^{36}$, including $n$-alkanes for retention index calculation. The derivatized samples $(1 \mu \mathrm{L})$ were analyzed by splitless- and 1:30 split-injection modes with a $6890 \mathrm{~N}$ gas chromatograph (Agilent, Santa Clara, CA, USA) connected to a Pegasus III time-of-flight mass spectrometer (Leco Instruments, St. Joseph, MI, USA). Metabolite annotation was performed by matching of mass spectra and retention index information to the Golm Metabolome Database using TagFinder software $\mathrm{s}^{36-38}$.

\section{Measurement of sugar content using HPAEC-PAD analysis}

A freeze-dried sample (15 mg, at least three replicates) was extracted in $1 \mathrm{~mL}$ of $80 \%$ methanol, with 0.3 mg of cellobiose (Wako, Osaka, Japan) as an internal standard for calibration, and incubated at $92^{\circ} \mathrm{C}$ for 10 minutes. The extraction step was repeated twice. The supernatant was transferred to a new tube and evaporated with CentriVap Centrifugal Vacuum Concentrators. The pellet was dissolved in $300 \mu \mathrm{L}$ of ultrapure (UP) water, 50 -fold diluted, and filtered through a $0.22-\mu \mathrm{m}$ syringe filter for further analysis.

For HPAEC-PAD, Dionex ICS 5000 ion chromatography system (Dionex, Sunnyvale, CA, USA) was used with a Carbo PacTM PA1 high-performance anion-exchange column $(4 \times 250 \mathrm{~mm})$ (Dionex). The elution buffer system was consisted of $150 \mathrm{mM} \mathrm{NaOH}$ (buffer A) and $500 \mathrm{mM} \mathrm{CH} 3 \mathrm{COONa}$ (Kemaus, Australia) in $150 \mathrm{mM} \mathrm{NaOH}$ (buffer B). The column was equilibrated by buffer $A$ with flow rate $1 \mathrm{~mL} / \mathrm{minute}$ at 30 ${ }^{\circ} \mathrm{C}$. The samples were eluted by multistep-gradients as following; constant $100 \%$ buffer $\mathrm{A}$ at $0-5$ minutes, linear gradient to $5 \%$ buffer $B$ at 5-8 minutes and constant at $5 \%$ buffer $B$ to 12 minutes, linear gradient to $20 \%$ buffer $B$ at $12-15$ minutes, linear gradient to $100 \%$ buffer $B$ at $15-17$ minutes and hold at $100 \%$ buffer $B$ for 2 min, and linear gradient to $100 \%$ buffer $A$ at $19-20$ minutes.

Quantification of the targeted sugars, including myo-inositol (Phytotechnology Laboratories, Lenexa, KS, USA), galactinol (TCl, Tokyo, Japan), sorbitol (Acros, Antwerp, Belgium), fructose (Sigma), glucose (Univar, Downers Grove, USA), 1-kestose (Wako), maltose (Condalab, Madrid, Spain), raffinose (TCI), and sucrose (Univar), was conducted using mixtures of the reference standards in the ranges of $0.1-2.5$ $\mathrm{mg} / \mathrm{mL}$.

\section{RNA extraction and transcriptome analysis}

Total RNA was extracted (in triplicate) from the samples at unripe and ripe stages, using the PureLink Plant RNA Reagent (Invitrogen Carlsbad, CA, USA) as per the manufacturer's instructions. cDNA was synthesized using the RevertAid First Strand cDNA Synthesis Kit (Thermo Fisher Scientific Inc., Waltham, MA, USA) according to the manufacturer's instructions. Next, transcriptome sequencing was performed using the BGISEQ-500 platform (BGI, Shenzhen, China). We used the OmicsBox program (v1.4.1.1) for transcriptome data analysis. Filtering of raw reads was performed, which resulted in paired-end clean reads, and unpaired reads that lost their corresponding sequence partners due to quality control 
procedures. Then, the de novo transcriptome assembly of the cleaned reads was performed using the Trinity (v2.8.5) package of the program with default parameters. The assembled transcripts were annotated with NCBI BLAST against non-redundant protein sequences (Nr v5), InterProScan, Gene Ontology (GO), and Kyoto Encyclopedia of Genes and Genomes (KEGG). The clean reads were mapped back to the assembled transcripts using the Bowtie2 (v2.3.5.1) package with default parameters, and the expression levels of the transcripts were quantified using the RSEM (v1.3.1) package with default parameters. The read counts were normalized using the reads per kilobase of transcript and per million mapped reads (RPKM) method. Pairwise differential expression analysis was performed using the Exact test (FDR < 0.05 ) of the software package edgeR (v3.28.0), when change in expression was 2-fold or higher. The differentially expressed genes (DEGs) were classified using GO classification and into specific biological pathways using KEGG.

\section{Reverse transcription quantitative polymerase chain reaction (RT-qPCR) analysis}

cDNA was used for gene expression analysis with RT-qPCR; the reaction mix was prepared using the Luna ${ }^{\circledR}$ Universal qPCR Master Mix (New England Biolabs, Ipswich, MA, USA) according to the manufacturer's protocol and the reaction was run on a CFX Real-Time PCR system (Bio-Rad Laboratories, Inc., Hercules, MA, USA). Relative expression levels were calculated using the $2^{-\Delta \Delta C T}$ method $^{39}$, based on the ratio of the expression of candidate genes and elongation factor 1 alpha (EF1a), a housekeeping gene $^{10}$. The primers used for RT-qPCR analyses are listed in Supplementary Table S8.

\section{Statistical analysis}

The metabolite data were processed by algorithms in MetaboAnalyst 4.0 (https://www.metaboanalyst.ca/) ${ }^{40}$. Principal component analysis (PCA), which is a dimension-reduction tool, was applied to investigate the relationships among samples. A volcano plot was used to select significant metabolites, which were designated as those with a relative intensity greater than the threshold ( $\mid \log 2$ (fold change $[\mathrm{FC}]) \mid>1, P<0.05$ ) between the two stages. Metabolite pathway analysis (MetPA) was performed to enrich the metabolite set into the relevant pathways. Pathways that reached cut-off values $(P<0.05$, pathway impact $\geq 0.1)$ were considered as perturbed pathways ${ }^{41}$. One-way ANOVA followed by Tukey's test in GraphPad Prism version 5 was used to compare the means of each sugar at different ripening stages $(P<0.05)$.

\section{Declarations}

\section{Data availability}

The raw sequences have been deposited into NCBI Sequence Read Archive (SRA) under the project accession number PRJNA683229 and CNGB Sequence Archive (CNSA) of China National GeneBank DataBase (CNGBdb) with project accession number CNP0001432. 


\section{Acknowledgements}

This work was supported by a Ratchadapisek Sompoch Grant, Chulalongkorn University (CUGR_62_10_23_04) and by Chulalongkorn University Fund (GRU 6203023003-1) (to S.S.), and the $90^{\text {th }}$ Anniversary of Chulalongkorn University (Ratchadaphisek Somphot Endowment Fund) (to S.S. and L.S.). L.S. was financially supported by the Development and Promotion of Science and Technology Talents Project (DPST) scholarship. We thank Miss Kittiya Tantisuwanichkul for assistance with RNA extraction for the durian sample. A.E., M.W., T.T., J.K., and R.H. were funded by the Max Planck Society.

\section{Conflict of interest}

The authors have no known conflict of interest to disclose.

\section{Contributions}

S.S. conceived the original screening and research plan. L.S. wrote the first draft of the manuscript. A.O. and R.S. performed CE-TOF/MS analysis. A.E. and J.K. performed GC-TOF/MS analysis. L.S. and K.W. performed HPAEC-PAD analysis. L.S. performed de novo transcriptome analysis. L.S. and P.P. analyzed the data. L.S., G.K., P.P., A.O., A.E., J.K., R.H., K.W., M.W., T.T., K.S. and S.S. revised the manuscript. All authors read and approved the final manuscript.

\section{References}

1. Charoenkiatkul, S., Thiyajai, P. \& Judprasong, K. Nutrients and bioactive compounds in popular and indigenous durian (Durio zibethinus Murr.). Food Chem. 193, 181-186 (2016).

2. Aziz, N. A. A. \& Jalil, A. M. M. Bioactive compounds, nutritional value, and potential health benefits of indigenous durian (Durio zibethinus Murr.): A review. Foods. 8, 96 (2019).

3. Gao, J., Zhang, Y., Li, Z. \& Liu, M. Role of ethylene response factors (ERFs) in fruit ripening. J. Food. Qual. 4, 15-20 (2020).

4. Tieman, D. M. et al. Identification of loci affecting flavour volatile emissions in tomato 
fruits. J. Ext. Bot. 57, 887-896 (2006).

5. Lombardo, V. A. et al. Metabolic profiling during peach fruit development and ripening reveals the metabolic networks that underpin each developmental stage. Plant Physiol. $157,1696-1710$ (2011).

6. Zhu, G. et al. Rewiring of the fruit metabolome in tomato breeding. Cell. 172, 249-261 (2018).

7. Pinsorn, P. et al. Metabolic variation in the pulps of two durian cultivars: Unraveling the metabolites that contribute to the flavor. Food Chem. 268, 118-125 (2018).

8. Teh, B. T. et al. The draft genome of tropical fruit durian (Durio zibethinus). Nat. Genet. 49, 1633-1641 (2017).

9. Khaksar, G., Sangchay, W., Pinsorn, P., Sangpong, L. \& Sirikantaramas, S. Genome wide analysis of the Dof gene family in durian reveals fruit ripening-associated and cultivar-dependent Dof transcription factors. Sci. Rep. 9, 12109 (2019).

10. Khaksar, G. \& Sirikantaramas, S. Auxin response factor $2 A$ is part of the regulatory network mediating fruit ripening through auxin-ethylene crosstalk in durian. Front. Plant Sci. 11, 543747 (2020).

11. Carrari, F. \& Fernie A. R. Metabolic regulation underlying tomato fruit development. J. Exp. Bot. 57, 1883-1897 (2006).

12. Füzfai, Z., Katona, Z. F., Kovács, E. \& Molnár-Perl, I. Simultaneous identification and quantification of the sugar, sugar alcohol, and carboxylic acid contents of sour cherry, apple, and berry fruits, as their trimethylsilyl derivatives, by gas chromatography-mass spectrometry. J. Agric. Food Chem. 52, 7444-7452 (2004).

13. Yamaki, S. Metabolism and accumulation of sugars translocated to fruit and their regulation. J. Jpn. Soc. Hortic. Sci. 79, 1-15 (2010). 
14. Jovanovic-Malinovska, R., Kuzmanova, S. \& Winkelhausen, E. Oligosaccharide profile in fruits and vegetables as sources of prebiotics and functional foods. Int. J. Food Prop. 17, 949-965 (2014).

15. Fichtner, F. et al. The role of trehalose 6-phosphate in shoot branching - local and nonlocal effects on axillary bud outgrowth in Arabidopsis rosettes. Preprint at https://doi.org/10.1101/2020.06.18.158568 (2020).

16. Yi, T. X. et al. Postharvest quality indices of different durian clones at ripening stage and their volatile organic compounds. Sci. Hortic. 264, 109169 (2020).

17. Roze, L. V. et al. Volatile profiling reveals intracellular metabolic changes in Aspergillus parasiticus: VeA regulates branched chain amino acid and ethanol metabolism. BMC Biochem. 11, 33 (2010).

18. Gonda, I. et al. Branched-chain and aromatic amino acid catabolism into aroma volatiles in Cucumis melo L. fruit. J. Exp. Bot. 61, 1111-1123 (2010).

19. Li, J., Schieberle, P. \& Steinhaus, M. Characterization of the major odor-active compounds in Thai durian (Durio zibethinus L. 'Monthong') by aroma extract dilution analysis and headspace gas chromatography-olfactometry. J. Agric. Food Chem. 60, 11253-11262 (2012).

20. Haruenkit, R. et al. Comparison of bioactive compounds, antioxidant and antiproliferative activities of Mon Thong durian during ripening. Food Chem. 118, 540547 (2010).

21. Sorrequieta, A., Ferraro, G., Boggio, S. B. \& Valle, E. M. Free amino acid production during tomato fruit ripening: a focus on L-glutamate. Amino Acids. 38, 1523-1532 (2010).

22. Sayed, O. M., Gammal, O. E. \& Salama, A. Effect of proline and tryptophan amino 
acids on yield and fruit quality of Manfalouty pomegranate variety. Sci. Hortic. 169, $1-5(2014)$.

23. Chen, D., Shao, Q., Yin, L., Younis, A. \& Zheng, B. Polyamine function in plants: Metabolism, regulation on development, and roles in abiotic stress responses. Front. Plant Sci. 9, 1945 (2019).

24. Tassoni, A., Watkins, C. B. \& Davies, P. J. Inhibition of the ethylene response by 1MCP in tomato suggests that polyamines are not involved in delaying ripening, but may moderate the rate of ripening or over-ripening. J. Exp. Bot. 57, 3313-3325 (2006).

25. Deewatthanawong, R., Nock, J. F. \& Watkins, C. B. Y-Aminobutyric acid (GABA) accumulation in four strawberry cultivars in response to elevated $\mathrm{CO}_{2}$ storage. Postharvest Biol. Technol. 57, 92-96 (2010).

26. Wu, X. et al. Gamma-aminobutyric acid (GABA) alleviates salt damage in tomato by modulating $\mathrm{Na}^{+}$uptake, the $G A D$ gene, amino acid synthesis and reactive oxygen species metabolism. BMC Plant Biol. 20, 465 (2020).

27. Brikis, C. J. et al. Targeted quantitative profiling of metabolites and gene transcripts associated with 4-aminobutyrate (GABA) in apple fruit stored under multiple abiotic stresses. Hortic. Res. 5, 61 (2018).

28. Chew, B. L. et al. The effect of adenosine monophosphate deaminase overexpression on the accumulation of umami-related metabolites in tomatoes. Plant Cell Rep. 36, 8187 (2016).

29. Ball, K. L., Green, J. H. \& Rees, T. Glycolysis at the climacteric of bananas. Eur. J. Biochem. 197, 265-269 (1991).

30. Yin, Y. et al. Metabolic alterations in organic acids and $\mathrm{Y}$-aminobutyric acid in developing tomato (Solanum lycopersicum L.) fruits. Plant Cell Physiol. 51, 1300-1314 
(2010).

31. Hua, X. J., Cotte, B. V., Montagu, M. V. \& Verbruggen, N. (1997). Developmental

regulation of pyrroline-5-carboxylate reductase gene expression in Arabidopsis. Plant

Physiol. 114, 1215-1224.

32. Hao, Y. Kitashiba, H., Honda., C., Nada, K. \& Moriguchi, T. Expression of arginine decarboxylase and ornithine decarboxylase genes in apple cells and stressed shoots. J. Exp. Bot. 56, 1105-1115 (2005).

33. Maloney, G. S. et al. Characterization of the branched-chain amino acid aminotransferase enzyme family in tomato. Plant Physiol. 153, 925-936 (2010).

34. Kochevenko, A. et al. Catabolism of branched chain amino acids supports respiration but not volatile synthesis in tomato fruits. Mol. Plant. 5, 366-375 (2012).

35. Oikawa, A., Fujita, N., Horie, R., Saito, K. \& Tawaraya K. Solid-phase extraction for metabolomic analysis of high-salinity samples by capillary electrophoresis-mass spectrometry. J. Sep. Sci. 34, 1063-1068 (2011).

36. Erban, A. et al. in Plant Cold Acclimation: Methods in Molecular Biology (Methods and Protocols) 2nd edn, Vol. 2156 (eds Hincha, D.K. \& Zuther, E.) Ch. 15 (Humana, 2020).

37. Kopka, J. et al. GMD@CSB.DB: the Golm Metabolome Database. Bioinformatics. 21, $1635-1638$ (2005).

38. Luedemann, A., Strassburg, K., Erban, A. \& Kopka, J. TagFinder for the quantitative analysis of gas chromatography - mass spectrometry (GC-MS) based metabolite profiling experiments. Bioinformatics. 24, $732-737$ (2008).

39. Livak, K. J. \& Schmittgen, T. D. Analysis of relative gene expression data using real time quantitative PCR and the $2^{-\Delta \Delta C t}$ method. Methods. 25, $402-408$ (2001). 
40. Pang, Z., Chong, J., Li, S. \& Xia, J. (2020). MetaboAnalystR 3.0: Toward an optimized workflow for global metabolomics. Metabolites. 10, 186.

41. Guo, Y. S. \& Tao, J. Z. Metabolomics and pathway analyses to characterize metabolic alterations in pregnant dairy cows on D 17 and D 45 after Al. Sci. Rep. 8, 5973 (2018).

\section{Figures}
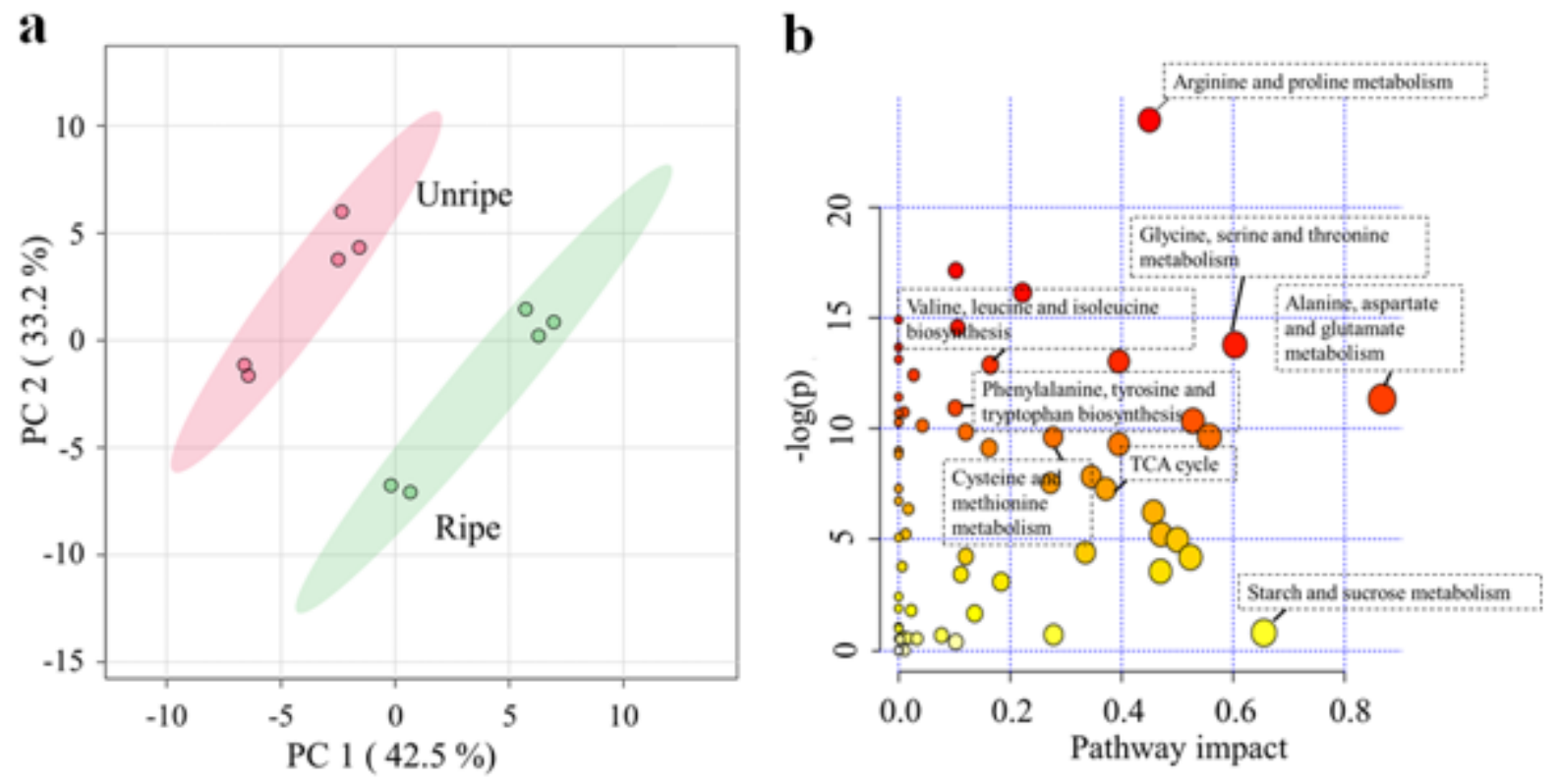

\section{Figure 1}

(a) Principal component analysis (PCA) score plot of metabolites in pulp of durian cv. Monthong at unripe and ripe stages, which are labelled in red and green, respectively. Each dot represents each biological replicate sample, and the circle represents $95 \%$ confidence intervals. (b) Metabolic pathway analysis (MetPA). Matched pathways related to ripening of durian cv. Monthong are displayed as circles. Color and size of the circles represent the $P$ value and pathway impact value, respectively. The focused pathways associated to our detected primary metabolites were labeled. Metabolic pathways with Pathway Impact $>0.1$ and $\mathrm{P}<0.05$ were perturbed pathways. 


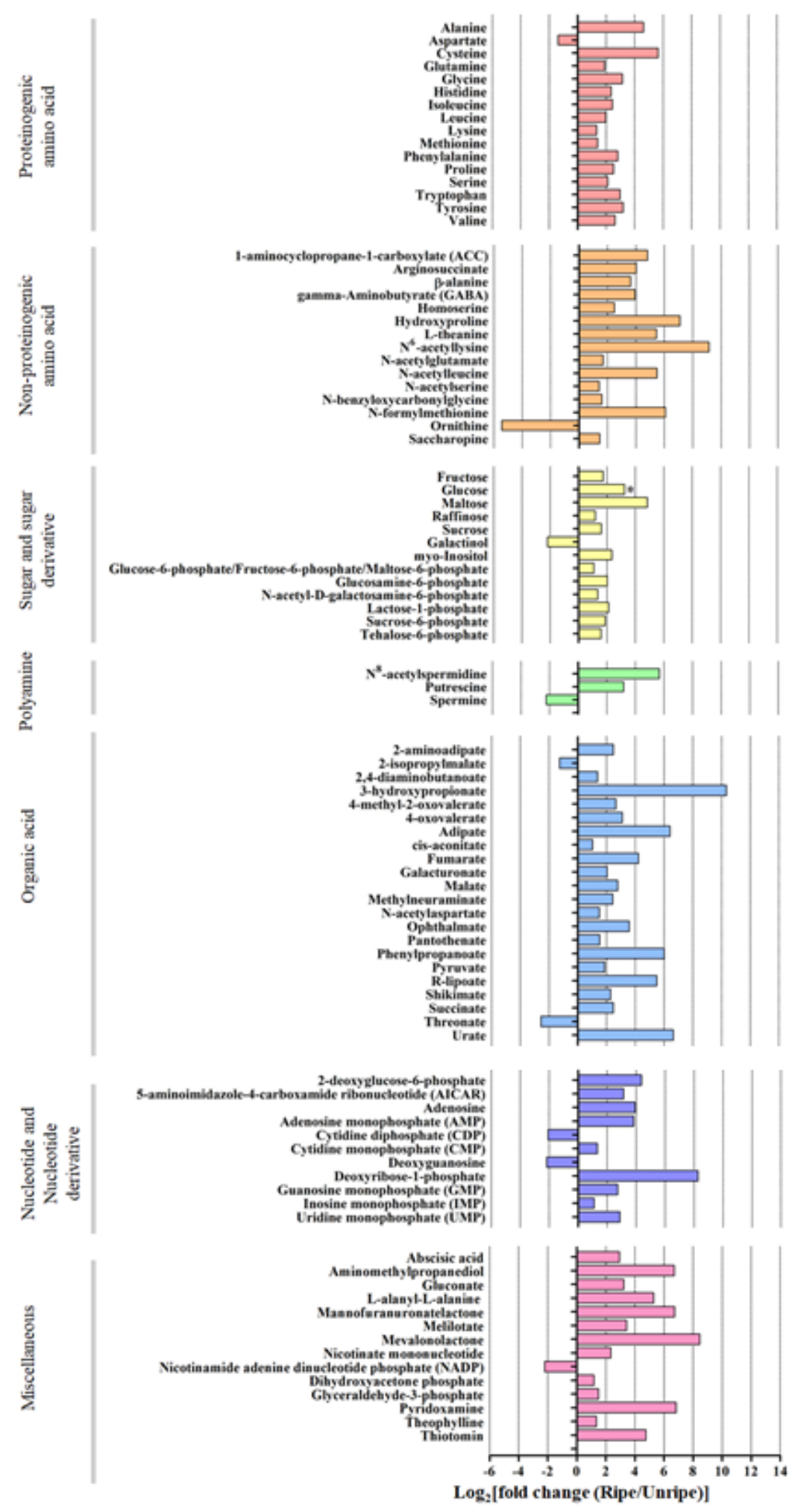

Figure 2

Log2 of fold change (F.C.) of metabolite intensity from ripe and unripe stages of durian cv. Monthong. Based on volcano plot $(|\log 2(\mathrm{FC})|>1, \mathrm{P}<0.05)$, these metabolites significantly changed during ripening process. Changes are represented in bar chart, indicating the increased levels (positive values) or decreased levels (negative values) of the metabolite intensity. ( ${ }^{*}=$ glucose which is significant under $\mathrm{P}=$ $0.1)$. 

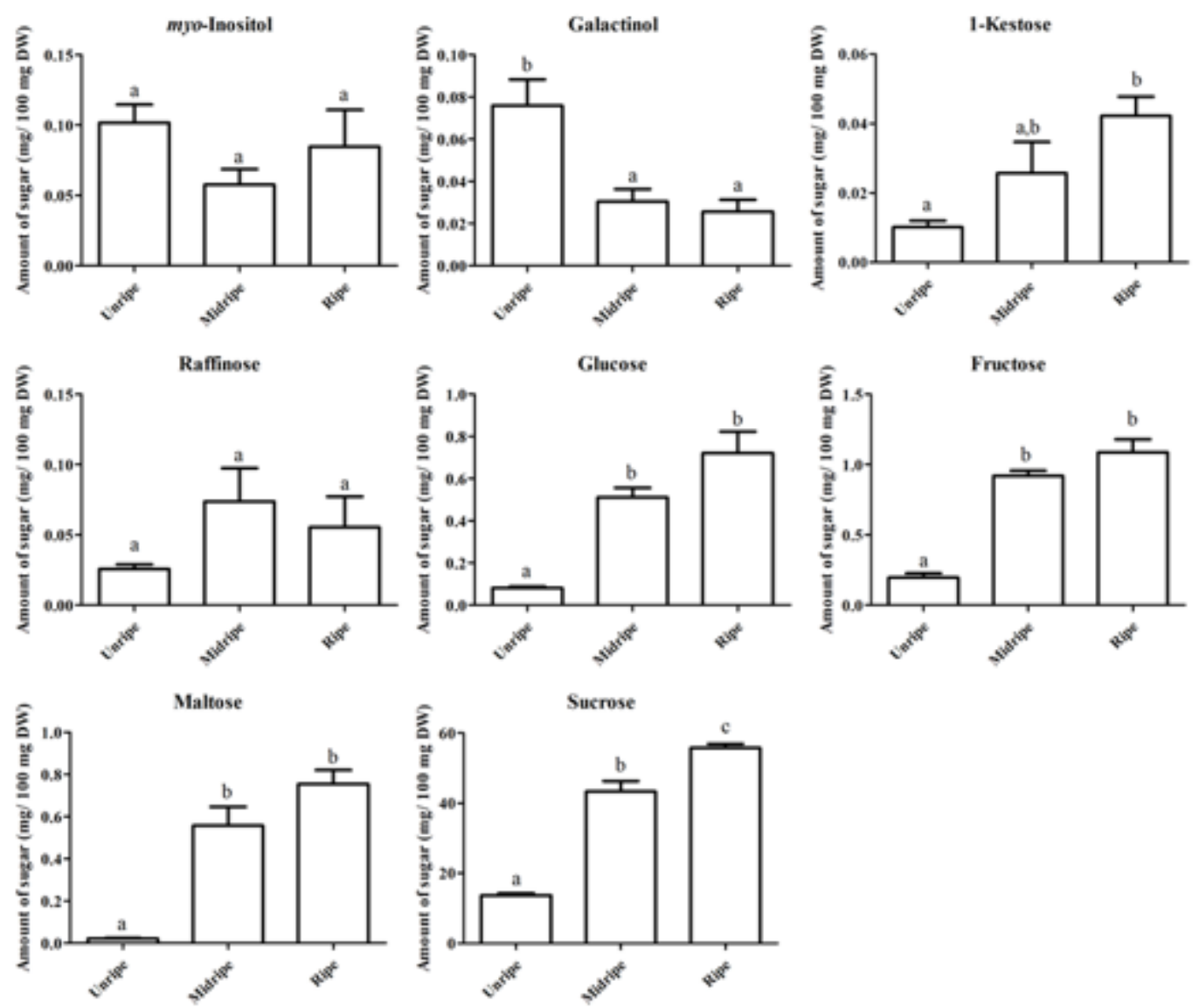

\section{Figure 3}

Soluble sugar content based on dry weight, including myo-inositol, galactinol, 1-kestose, raffinose, glucose, fructose, maltose, and sucrose contents (mg/100 mg dry weight (DW)) of the durian pulp at three ripening stages (including unripe, midripe, and ripe). Each bar indicates mean \pm standard error $( \pm$ SEM) values from triplicate experiments.

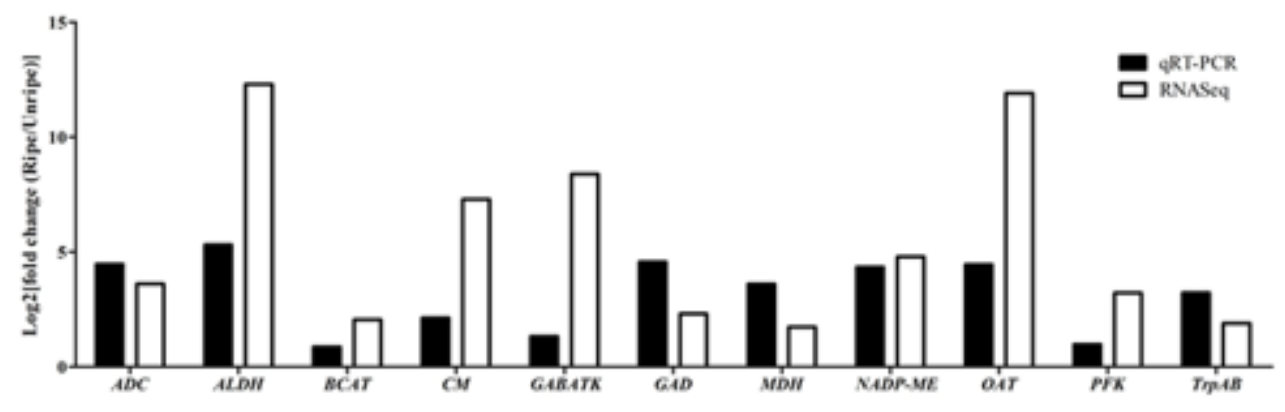

Figure 4

Log2 fold change in expression values in the ripe pulps relative to unripe pulps, as measured with RNAseq and RT-qPCR in the 11 selected genes. 


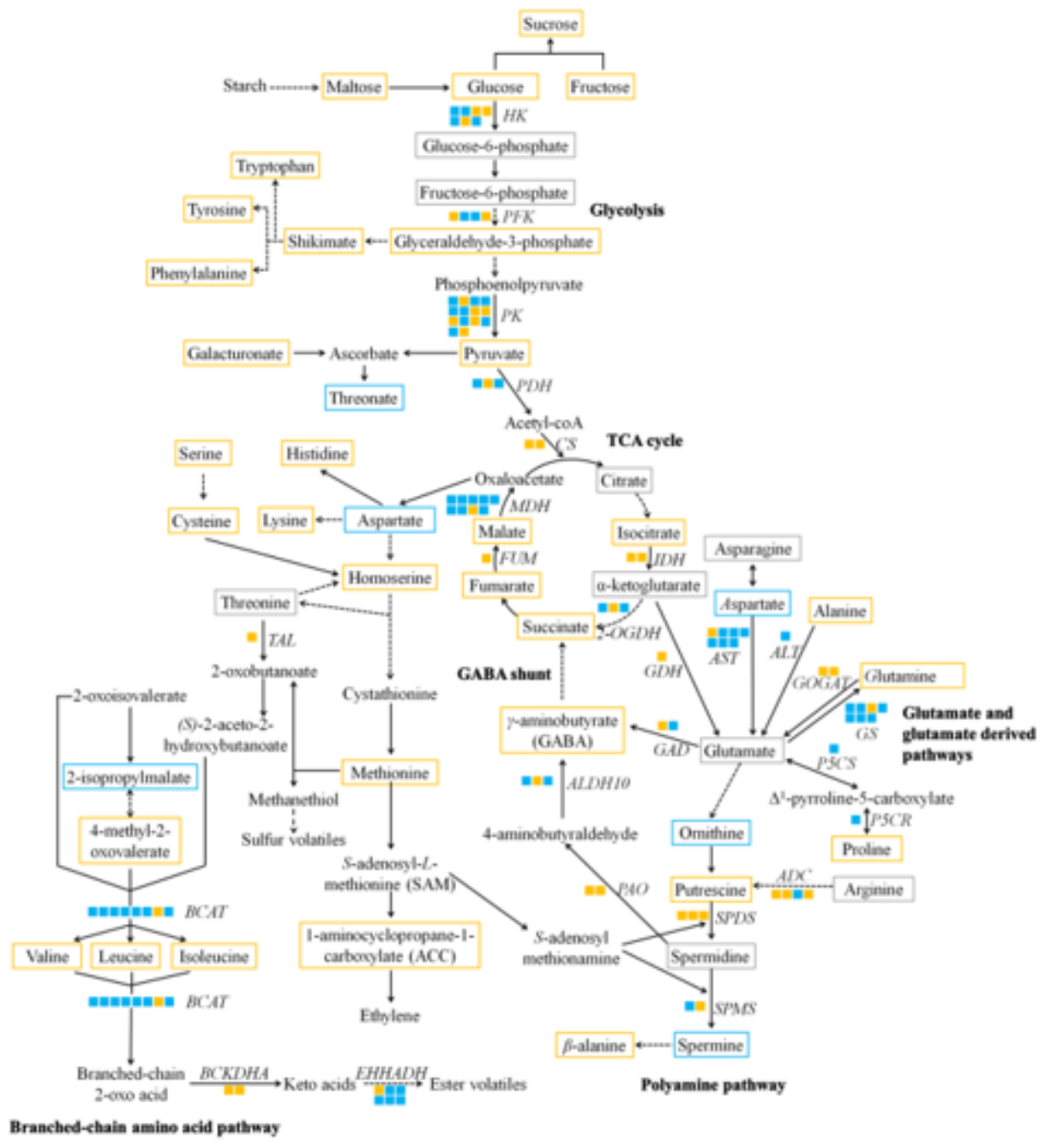

Figure 5

A metabolic map of significantly altered metabolites and DEGs of flavor-associated pathways during durian cv. Monthong ripening. Metabolites with significantly increased, significantly decreased, and unchanged contents are outlined in orange, blue, and grey, respectively. Undetectable metabolites are shown without boxes. Isoforms of each gene are represented by a solid box, and each isoform is arranged by locus number. Upregulated and downregulated isoforms are colored in orange and blue, respectively. HK, hexokinase; PFK, phosphofructokinase; PK, pyruvate kinase; PDH, pyruvate dehydrogenase; $\mathrm{CS}$, citrate synthase; IDH, isocitrate dehydrogenase; 2-OGDH, a-ketoglutarate dehydrogenase; FUM, fumarase; $\mathrm{MDH}$, malate dehydrogenase; AST, aspartate aminotransferase; ALT, alanine aminotransferase; GDH, glutamate dehydrogenase; GOGAT, glutamate synthase; GS, glutamine synthetase; P5CS, $\triangle 1$-pyrroline-5-carboxylate synthetase; P5CR, $\triangle 1$-pyrroline-5-carboxylate reductase; $A D C$, arginine decarboxylase; SPDS, spermidine synthase; SPMS, spermine synthase; GAD, glutamate decarboxylase; PAO, polyamine oxidase; ALDH10, 4-aminobutanal dehydrogenase; TAL, threonine ammonia-lyase; BCAT, branched-chain amino acid transaminase; BCKDHA, 3-methyl-2-oxobutanoate dehydrogenase; EHHADH, long-chain-enoyl-CoA hydratase. 


\section{Supplementary Files}

This is a list of supplementary files associated with this preprint. Click to download.

- SupplementaryTable.xlsx

- Supplementaryfigure.pptx 